\title{
The Calculation of Cation Distributions in Oxide Scales by an Approximate Analytical Method
}

\author{
By D. P. Whittle*, B. D. Bastow** and G. C. Wood**
}

\begin{abstract}
The differential equations which describe the distribution of cations in solid solution oxide scales are re-examined. It is shown that an approximate analytical solution can be derived in terms of a finite series providing the point defects responsible for diffusion carry an integral excess charge relative to the cation lattice. If the charge is non-integral the series becomes infinite, but convergent. The series approximation is applicable to any scale composition and is compared to an alternative, error function, expression, derived by previous authors, which is restricted in its application to dilute solutions. An upper limiting composition for the application of this latter expression is defined and a modification which overcomes this difficulty is suggested. The relationships are applied to a description of nickel distributions in scales on $\mathrm{Ni}-\mathrm{Co}$ alloys.
\end{abstract}

(Received July 22, 1976)

\section{Introduction}

There have been a number of recent attempts to analyze binary alloy oxidation for those systems where a single phase scale containing both alloy components is formed using the theoretical analysis developed by Wagner ${ }^{(1)}$, and modified by Coates and Dalvi $^{(2)}$. These latter authors ${ }^{(3)}$ analyzed the cation distribution ${ }^{(4)}$ in the (CoNi)O scale formed on a Ni-10.9\% Co alloy and Smeltzer et $a .^{(5)(6)}$ calculated the distributions of $\mathrm{Fe}$ cations in the oxide scales formed on $\mathrm{Co}-\mathrm{Fe}$ and $\mathrm{Ni}-\mathrm{Fe}$ alloys. More recently, Japanese workers have related experimentally determined cation distributions in scales formed on Co-rich, Co-Ni alloys with theoretically calculated profiles $^{(7)}$. However, in all these cases it was necessary to use data from the experimentally measured profiles, together with the experimental value for the parabolic rate constant, in order to establish the boundary conditions for the calculation of the theoretical cation profile. Consequently, the agreement between theoretical and experimental profiles

* Department of Metallurgy and Materials Science, University of Liverpool, P.O. Box 147, Liverpool L69 3BX, England.

** Corrosion and Protection Centre, University of Manchester Institute of Science and Technology, P.O. Box 88, Manchester M60 1QD, England. does not represent a complete test of the validity of the model.

Whittle et al. ${ }^{(8)}$ showed that provided the rate of interdiffusion within the alloy was negligible in comparison to the transport rates in the scale layers, as is the case in the systems referred to above, the gradient of the cation distribution within the scale at the alloy/scale interface approached zero. As a result the composition of the scale at the alloy/scale interface could be calculated from the ratio of cation diffusivities in the scale. Combining this boundary value with a thermodynamic description of the exchange reaction between alloy and scale provides a value of the oxygen activity at the alloy/scale interface; the oxygen activity at the scale/gas interface is defined by the composition of the oxidizing gas. These three boundary values enabled a complete solution to the theoretical equations to be obtained ${ }^{(9)}$, which was completely independent of the experimental profiles. The resulting agreement ${ }^{(9)}$ between theoretical and experimental profiles for a number of Ni-Co alloys provides strong support for the Wagner model. More recent work ${ }^{(10)}$ has shown the dependence of cation concentration profiles and overall scaling rate on the various parameters involved, including the relative diffusion rates of participating metals in the alloy and ions in the scale, the degree of selective oxidation of the appropriate alloy component, the rates of diffusion in the scale, 
and the activity of the oxidizing atmosphere. Results are presented for a wide range of systems.

Determination of the cation profiles and oxygen activity in growing scales requires the simultaneous solution of second and first order differential equations. A further complication arises because the cation diffusion coefficients are functions of both cation concentration ratio and oxygen activity and precise solutions of the equations have only been obtained using numerical integration procedures. Wagner ${ }^{(1)}$ presented an approximate solution for the particular case of the sulphide scale formed on $\mathrm{Ag}-\mathrm{Cu}$ alloys, where because of the high disorder in the $\mathrm{Ag}_{2} \mathrm{~S}$ $\mathrm{Cu}_{2} \mathrm{~S}$ scale, variation of cation diffusivities with sulphur activity and composition can be safely ignored. Narita et al. ${ }^{(1)}$ used a similar approximation for sulphide scales formed on $\mathrm{Fe}-\mathrm{Ni}$ alloys. The same authors ${ }^{(7)}$ also tried a similar approximate solution in analyzing cation distributions in Co-rich oxide scales formed on $\mathrm{Co}-\mathrm{Ni}$ alloys. However, whilst the relatively low $\mathrm{NiO}$ content of the scales means that the dependence of cation diffusivities on scale composition can be ignored, a strong dependence on oxygen activity remains, and this markedly limited the applicability of their approximate solution. The present paper attempts to provide a better approximate solution, and whilst it is realized that more exact solutions are easily obtainable by numerical analysis techniques, the advantage of the analytical expression produced here is that it can be used in more complex systems involving solid solution scale growth, such as interface stability problems ${ }^{(12)}$, or where multilayered scale growth is considered.

\section{Theoretical Equations}

According to Wagner ${ }^{(1)}$, the relationship between the rate constant for growth of a mono-phase scale on a binary alloy and the distribution of metal cation concentration and non-metal activity in the scale is given by the following two interrelated equations (eqs. (11) and (19) of reference (1)):

$$
\begin{gathered}
D_{A}(1-\xi)\left[-\frac{\partial \ln a_{A(X)}}{\partial \xi} \frac{d \xi}{d y}+\frac{Z_{A}}{Z_{X}} \frac{d \ln a_{X}}{d y}\right] \\
+D_{B} \xi\left[-\frac{\partial \ln a_{B(X)}}{\partial \xi} \frac{d \xi}{d y}+\frac{Z_{B}}{Z_{X}} \frac{d \ln a_{X}}{d y}\right]=k, \quad(1) \\
y k \frac{d \xi}{d y}=-\frac{d}{d y}\left[D_{B} \xi\left(\frac{\partial \ln a_{B(X)}}{\partial \xi} \frac{d \xi}{d y}-\frac{Z_{B}}{Z_{X}} \frac{d \ln a_{X}}{d y}\right)\right] .
\end{gathered}
$$

$D_{A}, D_{B}$ self-diffusivities of metal cations $A$ and $B$ respectively in the oxide scale;

$\xi$ local equivalent fraction of compound $B(X)$ in the scale;

$a_{A(X)}, a_{B(X)}$ and $a_{X}$ activities of compounds $A(X)$ and $B(X)$ and non-metal $X$ in the scale;

$y$ fractional distance across the scale; $Z_{A}, Z_{B}$ and $Z_{X}$ valencies of $A, B$ and nonmetal $X$ respectively.

In order to obtain solutions to eqs. (1) and (2), it is appropriate to introduce a number of further simplifications. Firstly, it is assumed that the solid solution $A(X)-(B X)$ shows ideal behaviour, when

$$
\frac{\partial \ln a_{A(X)}}{\partial \xi}=-\frac{1}{1-\xi} ; \frac{\partial \ln a_{B(X)}}{\partial \xi}=\frac{1}{\xi} .
$$

Furthermore, for convenience the valencies of the two cations and the non-metal are made equal:

$$
Z_{A}=Z_{B}=\left|Z_{X}\right| \text {. }
$$

As indicated earlier, the cation diffusion coefficients, $D_{A}$ and $D_{B}$, depend essentially on the concentration of cation vacancies in the scale, which in turn depend on the composition and on the non-metal activity. As shown previously ${ }^{(2)(9)}$ this is best expressed by

$$
D_{B}=D_{B}^{0} \beta^{\xi-1} a_{X}^{1 / \nu} ; D_{A}=p D_{B}
$$

where $\beta=N_{B(X)}^{\alpha} / N_{A(X)}^{\alpha}$, the ratio of vacancy mole fractions in the pure compounds $B(X)$ and $A(X), p$ is essentially the ratio of jump frequencies of cations $A$ and $B$ into an adjacent cation vacancy, $D_{B}^{0}$ is the diffusivity of cations in pure $B(X)$ at unit activity of $X$, and $1 / v$ is the exponent in the dependence of cation vacancy concentration on non-metal activity.

Wagner and Narita et al., in developing their approximate solutions to eqs. (1) and (2) neglected any dependence of $D_{A}$ and $D_{B}$ on 
either $\xi$ or $a_{X}$, and whilst this is justified in the sulphide scales referred to by Wagner ${ }^{(1)}$, it represents a severe limitation for corresponding oxides $^{(7)}$. Here, the non-metal activity dependence is described completely, but detailed concentration dependence $(\xi)$ is neglected. However, an estimate of the average prevailing cation diffusivity in a scale is incorporated as a constant term by substituting $\xi=\xi_{a v}$ in eq. (5), where $\xi_{a v}$, the average value of $\xi$ in the scale, is defined by

$$
\xi_{a v}=\int_{0}^{1} \xi d y
$$

Thus, eq. (5) becomes:

$$
D_{B} \cong D_{B}^{a v} a_{X}^{1 / v} ; D_{A}=p D_{B}
$$

where $D_{B}^{a v}=D_{B}^{0} \beta^{\xi_{a v}-1}$.

It is also appropriate to convert the rate constant $k$ to dimensionless form:

$$
k^{*}=k / D_{B}^{a v} \text {. }
$$

Substitution of eqs. (3), (4), (7) and (8) into eq. (1) leads to

$$
(p-1) \frac{d \xi}{d y}+[p(1-\xi)+\xi] \frac{d \ln a_{X}}{d y}=\frac{k^{*}}{a_{X}^{1 / v}} .
$$

Following the precise solutions of eqs. (1) and (2) given earlier ${ }^{(9)(10)}$, the rate constant $k^{*}$ is not strongly affected by $d \xi / d y$, and the first term in eq. (9) can be neglected. Furthermore, $\xi_{a v}$ can replace $\xi$ in the second term, giving

$$
a_{X}^{1 / v} d \ln a_{X}=\frac{k^{*}}{p\left(1-\xi_{a v}\right)+\xi_{a v}} d y .
$$

Integration of eq. (10) using $a_{X}=a_{X}^{\prime}$ at $y=0$ as the lower limit, where $a_{X}^{\prime}$ is the non-metal activity at the alloy/scale interface, gives

$$
v\left[\left(a_{X}\right)^{1 / v}-\left(a_{X}^{\prime}\right)^{1 / v}\right]=\frac{k^{*} y}{p\left(1-\xi_{a v}\right)+\xi_{a v}} .
$$

In most of the scale

$$
a_{X}^{\prime} \ll a_{X}
$$

and can be neglected: Thus

$$
\left(a_{X}\right)^{1 / v}=\frac{k^{*} y}{v\left[p\left(1-\xi_{a v}\right)+\xi_{a v}\right]}
$$

which in substitution in eq. (10) gives, after re-arrangement

$$
\frac{d \ln a_{\mathrm{x}}}{d y}=\frac{v}{y} .
$$

Furthermore, since $a_{X}=a_{X}^{\prime \prime}$ at $y=1$, where $a_{X}^{\prime \prime}$ is the non-metal activity at the scale/gas interface, eq. (11) reads

$$
k^{*}=v\left[p\left(1-\xi_{a v}\right)+\xi_{a v}\right]\left(a_{X}^{\prime \prime}\right)^{1 / v} \text {. }
$$

Substitution of eqs. (12), (13) and (14) along with (3), (4), (7) and (8) into (2) gives on rearrangement

$$
\frac{d^{2} \xi}{d y^{2}}=\frac{d \xi}{d y}\left[(v-1) \frac{1}{y}-v\left\{p\left(1-\xi_{a v}\right)+\xi_{a v}\right\}\right] .
$$

Integration of (15) using $(d \xi / d y)_{y=1}$ at $y=1$ as the lower limit gives

$$
\frac{d \xi}{d y}=\left(\frac{d \xi}{d y}\right)_{y=1} e^{v p^{*}} e^{-v p^{*} y} y^{(v-1)}
$$

with

$$
p^{*}=p\left(1-\xi_{a v}\right)+\xi_{a v} .
$$

If $v$ is a positive integer, which simple defect theory of the oxide predicts it should be, then eq. (16) can be integrated repeatedly by parts, to give

$$
\xi=\xi^{\prime}+\left(\frac{d \xi}{d y}\right)_{y=1} e^{v p^{*}}\left\{\frac{(v-1) !}{\left(v p^{*}\right)^{v}}-e^{-v p^{*} y} \sum_{r=1}^{r=v} \frac{v !}{v(v-r) !}\left(\frac{1}{v p^{*}}\right)^{r} y^{(v-r)}\right\}
$$

where $\xi=\xi^{\prime}$ at $y=0$ is the lower limit of integration.

Wagner's ${ }^{(1)}$ original mass balance at $y=1$ was given as

$$
-\left[D_{B} \xi\left\{\frac{\partial \ln a_{B(X)}}{\partial \xi} \frac{d \xi}{d y}-\frac{Z_{B}}{Z_{X}} \frac{d \ln a_{X}}{d y}\right\}\right]_{y=1}=\xi^{\prime \prime} k
$$

where $\xi^{\prime \prime}$ represents the value of $\xi$ at $y=1$. Using eqs. (3), (4), (7), (8), (12), (13), (14) and (17) this gives

$$
\left(\frac{d \xi}{d y}\right)_{y=1}=v \xi^{\prime \prime}\left(1-p^{*}\right)
$$


which on substitution in eq. (18) and putting $y=1$ when $\xi=\xi^{\prime \prime}$, yields

$$
\xi^{\prime \prime}=\frac{\xi^{\prime}}{1-\left(1-p^{*}\right) v !\left[\frac{e^{v p^{*}}}{\left(v p^{*}\right)^{v}}-\sum_{r=1}^{r=v} \frac{1}{(v-r) !}\left(\frac{1}{v p^{*}}\right)^{r}\right]}
$$

and

$$
\xi=\xi^{\prime}+\xi^{\prime \prime}\left(1-p^{*}\right) e^{v p^{*}}\left[\frac{v !}{\left(v p^{*}\right)^{v}}-e^{-v p^{*} y} \sum_{r=1}^{r=v} \frac{v !}{(v-r) !}\left(\frac{1}{v p^{*}}\right)^{r} y^{(v-r)}\right] .
$$

Under conditions of negligible diffusion in the alloy in comparison to the scale

$$
\xi_{a v}=N_{B}^{0}
$$

$N_{B}^{0}$ being the bulk mole fraction of component $B$ in the alloy. Using eq. (6) and the integration of eq. (22), $\xi^{\prime}$ can be found. However, the approximation given earlier ${ }^{(8)}$, namely

$$
\xi^{\prime}=\frac{p \xi_{a v}}{1+\xi_{a v}(p-1)}
$$

is more reliable. The series approximation, eq. (22) whilst reproducing closely the shape of the cation profile does not provide a good estimate of the value at $y=0$, except when $p$ is close to unity or $\xi_{a v}$ (and hence $N_{B}^{O}$ ) is either small or close to unity.

Equation (22) thus describes the cation profile through the scale, and eq. (13) the dimensionless parabolic rate constant; combining eqs. (13) and (14) gives the non-metal activity profile as

$$
a_{X}^{1 / v}=\left(a_{X}^{\prime \prime}\right)^{1 / v} y
$$

For non integral values of $v$, it is not possible to integrate eq. (16) to produce eq. (18); instead, a Taylor expansion is used for the second exponential term, giving

$$
\begin{aligned}
\frac{d \xi}{d y}= & \left(\frac{d \xi}{d y}\right)_{y=1} e^{v p^{*}} \sum_{r=1}^{\infty}(-1)^{r+1} \frac{1}{(r-1) !} \\
& \times\left(v p^{*}\right)^{r-1} y^{v+(r-2)} .
\end{aligned}
$$

This can then be integrated term by term, using $\xi=\xi^{\prime}$ at $y=0$ as the lower limit:

$$
\begin{aligned}
\xi= & \xi^{\prime}+\left(\frac{d \xi}{d y}\right)_{y=1} e^{v p^{*}} y^{v} \\
& \times \sum_{r=1}^{\infty} \frac{1}{v+(r-1)} \frac{1}{(r-1) !}\left(v p^{*} y\right)^{r-1}
\end{aligned}
$$

Again, using the mass balance eqs. (19) and (20), this gives

$$
\xi^{\prime \prime}=\frac{\xi^{\prime}}{1-\left(1-p^{*}\right) e^{v p^{*}} S(1)}
$$

and

$$
\xi=\xi^{\prime}+\xi^{\prime \prime}\left(1-p^{*}\right) e^{v p^{*}} y^{v} S(y)
$$

where $S(y)$ represents the summation in eq. (27), and has the specific values $S(1)$ for $y=1$. The rate of convergence of $S(y)$ varies with the relative values of the other parameters involved and must be determined in each case. However, it is anticipated that in the majority of cases, the finite series given in eq. (21) can be used without undue loss in accuracy, since the cation profile shape is relatively insensitive to the value of $v^{(9)}$.

\section{Discussion}

The set of eqs. (13), (21), (22) and (25) provides an approximate, but nevertheless, complete description of the growth rate of a solid solution scale and the cation concentration and anion activity distributions within the scale. The calculation of approximate values of $k^{*}$ from eq. (13) has been considered elsewhere for $\mathrm{Ni}-\mathrm{Co}$ alloys ${ }^{(9)}$, and the estimated values show good agreement with experimental data. In contrast, the approximation for $a_{X}(y)$, eq. (25), clearly becomes increasingly inaccurate as $y \rightarrow 0$, since it implies that $a_{X} \rightarrow 0$, whereas in fact the value of $a_{X}$ is finite at the alloy/scale interface. Since the limitations of these features of the analysis are well defined, only the reliability of the cation profiles calculated using the series approximation, eq. (22), is considered further.

\section{Analytical solutions for scales on dilute alloys}

The finite series solution derived in the present paper is compared to the error function 
solution derived by Wagner ${ }^{(1)}$ and used to analyze dilute $\mathrm{Co}-\mathrm{Ni}$ alloys by Narita and Nishida $^{(7)}$. This latter solution is written as:

where

$$
\begin{aligned}
\frac{\xi_{\mathrm{NiO}}}{N_{\mathrm{Ni}}^{0}}= & \frac{1}{P\left(1+C_{3}\right)}\left\{C_{3}-\frac{\pi^{1 / 2} g \exp C_{1}^{2}}{2 C_{2}}\right. \\
& \left.\times\left[\operatorname{erf} C_{1}-\operatorname{erf}\left(C_{1}-C_{2 y}\right)\right]\right\}
\end{aligned}
$$

$$
\begin{gathered}
P=D_{\mathrm{Ni}} / D_{\mathrm{Co}} \\
C_{1}=\left(\frac{1}{2} g P\right)^{1 / 2} \\
C_{2}=\left(\frac{1}{2} g / P\right)^{1 / 2} \\
C_{3}=\frac{P}{1-P} \exp [g(1-1 / 2 P)] \\
+\frac{\pi^{1 / 2} g}{2 C_{2}}\left[\operatorname{erf} C_{1}-\operatorname{erf}\left(C_{1}-C_{2}\right)\right] \exp C_{1}^{2} . \\
g=\frac{-\Delta G_{\mathrm{CoO}}^{0}}{R T}
\end{gathered}
$$

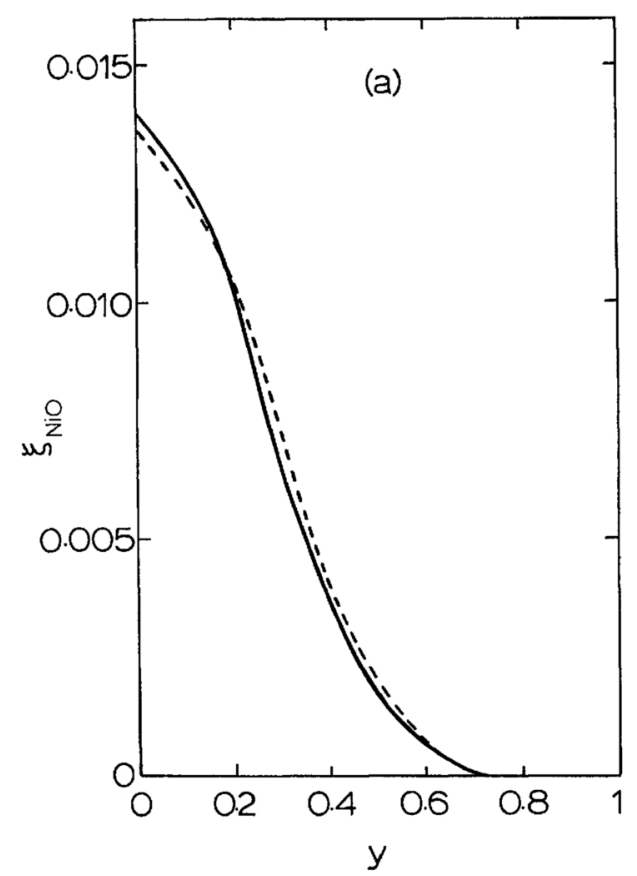

$\Delta G_{\mathrm{COO}}^{0}$ is the standard free energy of formation of $\mathrm{CoO}$ and $\xi_{\mathrm{NiO}}$ is the local equivalent fraction of $\mathrm{NiO}$ in the scale. Note that eq. (30) shows that the normalized cation profile in the scale is independent of the nickel concentration in the alloy.

Narita and Nishida ${ }^{(7)}$ presented results for the profiles in scales on alloys of Co containing $0.45 \% \mathrm{Ni}, 2.46 \% \mathrm{Ni}$ and $7.23 \% \mathrm{Ni}$ at $1200{ }^{\circ} \mathrm{C}$. The pertinent values of $P$ and $g$ they used to calculate the profiles using eq. (3) are $P=0.313$ and $g=10.63$. The equivalent data for using the series approximation solution presented in this work, are $p=3.19$ (the reciprocal of the 0.313 , since the roles of $\mathrm{Ni}$ and $\mathrm{Co}$ have been reversed) and $v=3$ (for diffusion of $\mathrm{Ni}^{++}$in $\left.\mathrm{NiO}^{(13)}\right)$. The nickel cation profiles in the scales formed on $\mathrm{Co}-0.45 \% \mathrm{Ni}$ and $\mathrm{Co}-7.23 \%$ $\mathrm{Ni}$ calculated from the error function approximation $^{(7)}$ and the series approximation, eq. (22), are shown in Fig. 1. It is noted that the value of $P$ used in the calculation of the error function profile is that chosen by Narita

Fig. 1 Ni profiles in scales formed at $1200^{\circ} \mathrm{C}$ on dilute $\mathrm{Co}-\mathrm{Ni}$ alloys containing (a) $0.45 \mathrm{wt} \%$ $\mathrm{Ni}$ and (b) $7.23 \mathrm{wt} \% \mathrm{Ni}$ calculated using the alternative approximations, eqs. (22) and (30). The profiles determined from the error function expression, eq. (32), are calculated using the data given in Ref. (7) which results in very close agreement with the experimental profile for the scale formed on $\mathrm{Co}-0.45 \% \mathrm{Ni}$. 
and Nishida ${ }^{(7)}$ to give optimum agreement with their experimental profile measured by microprobe analysis for the $\mathrm{Co}-0.45 \% \mathrm{Ni}$ scale. Consequently, since the series and error function approximations exhibit good agreement with each other for this dilute alloy, it follows that the series approximation also provides a good description of the experimental data.

However, although the profiles are substantially equivalent for the scales on the dilute alloys, it is noted that the discrepancy between the two curves does in fact increase as the concentration of nickel in the alloy (and hence the scale) increases. This arises because the error function solution is limited to the situation where $\xi \ll 1$; a similar restriction is not applicable to eq. (22) particularly when eq. (24) is used for $\xi^{\prime}$. Hence, it is expected that differences between the alternative approximations will continue to increase as the $\mathrm{Ni}$ concentration in the alloy, and thus $\xi_{a v}$ in the scale, is increased, making the error function approximation more inaccurate. The limiting composition, above which eq. (30) becomes meaningless, is readily obtained, since the value of $\xi$ cannot exceed unity at any point in the scale. Using the notation of eqs. (30)(34), when $D_{\mathrm{Ni}}<D_{\mathrm{Co}}, P<1$, and the maximum concentration of $\mathrm{Ni}$ in the scale occurs at the alloy/scale interface. Thus at $y=0$, the limiting bulk alloy composition for the applicability of eq. (30) is given by putting $\xi^{\prime}<1$, and

$$
N_{\mathrm{Ni}}^{0} \leq \frac{\left(1+C_{3}\right)_{P}}{C_{3}} .
$$

For the particular case considered above, where $p=0.313$ and $g=10.63, C_{3}$ has a value of 23.321 and thus the error function approximation is meaningless for alloys with $N_{\mathrm{Ni}}^{0}>0.326$. Clearly the limiting value will be different for other $P$ and $g$. However, this limitation could be obviated if eq. (24) for $\xi^{\prime}$ were incorporated into eq. (30). This would then read

$$
\begin{aligned}
\xi= & \xi^{\prime}-\left[\frac{\xi_{a v}}{p}-\xi^{\prime}\right] \frac{\pi^{1 / 2} g \exp C_{1}^{2}}{2 C_{2}} \\
& -\left[\operatorname{erf} C_{1}-\operatorname{erf}\left(C_{1}-C_{2} y\right)\right] .
\end{aligned}
$$

\section{Calculations for scales on concentrated alloys}

On nickel-rich alloys essentially similar solid solution scales are formed but there is also a limited extent of internal oxidation ${ }^{(14)}$. This is unlikely to have any significant effect on the cation concentration profiles, and these have been measured by the present authors ${ }^{(9)}$. The scales in this case were formed at $1000^{\circ} \mathrm{C}$ and the following pertinent data are required for the calculation of the cation profiles by the two approximate methods:

$$
\begin{aligned}
& \text { Error function approximation } \\
& \qquad P=0.2, g=13.68, \\
& \text { Series approximation } p=5, v=3 .
\end{aligned}
$$

Substitution of the relevant data in eq. (36) shows that the limiting alloy composition for the application of the error function approximation is $N_{\mathrm{Ni}}^{0}<0.213$. For alloys richer in nickel, only the series approximation can be used, and consequently the two approximations are compared for an alloy composition just below this limit, $N_{\mathrm{Ni}}^{0}=0.2$, in Fig. 2. They are also compared with an experimental profile measured by electron probe microanalysis, as described in an earlier paper ${ }^{(9)}$, and an accurate theoretical profile obtained from a solution of eqs. (1) and (2) using a finite difference technique ${ }^{(9)}$. This involves the calculation of the interdependent profiles of $\xi(y)$ and $a_{X}(y)$ by the simultaneous solution of eqs. (1) and (2), together with eq. (19), using an iterative procedure to satisfy the three independently defined boundary conditions: $\xi^{\prime}$ from eq. (24) and $a_{X}^{\prime}$ and $a_{X}^{\prime \prime}$ from auxiliary thermodynamic relations. The latter profile is in good agreement with the experimental profile except in the vicinity of the alloy/scale interface. The profile calculated from the series approximation, eq. (22), also reproduces the overall variation in $\xi_{\mathrm{NiO}}$ although not with the same precision as the finite difference solution.

Finally, while the error function approximation in its original form (eq. (30)) cannot be applied when $N_{\mathrm{Ni}}^{0}>0.213$, when the parameters have the particular values given above, the series approximation or the modified error 


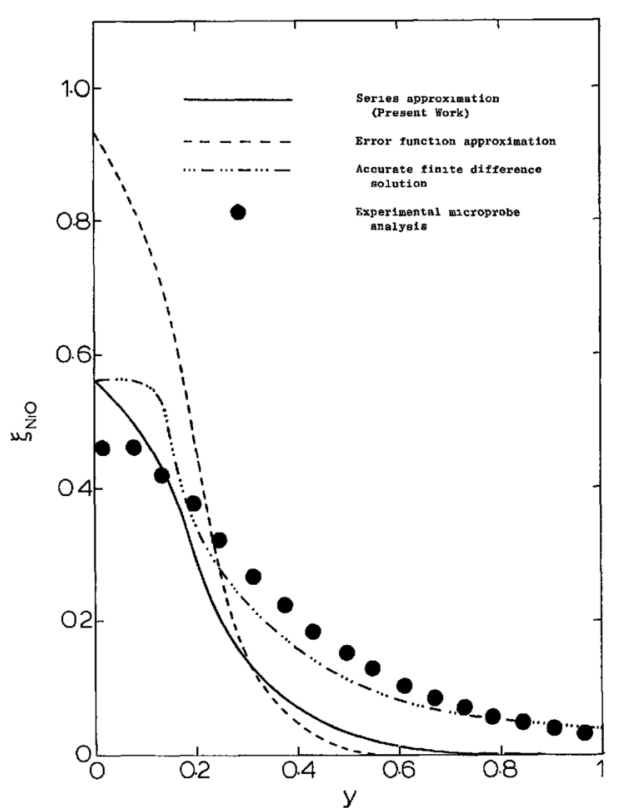

Fig. 2 A comparison between the experimental Ni profile in the scale formed on a $\mathrm{Co} 20 \mathrm{wt} \% \mathrm{Ni}$ alloy at $1000^{\circ} \mathrm{C}$ and theoretical profiles calculated from the finite series and error function approximations, eqs. (22) and (30) respectively, and from an accurate finite difference calculation. This alloy composition lies just within the limit of validity of the error function approximation.

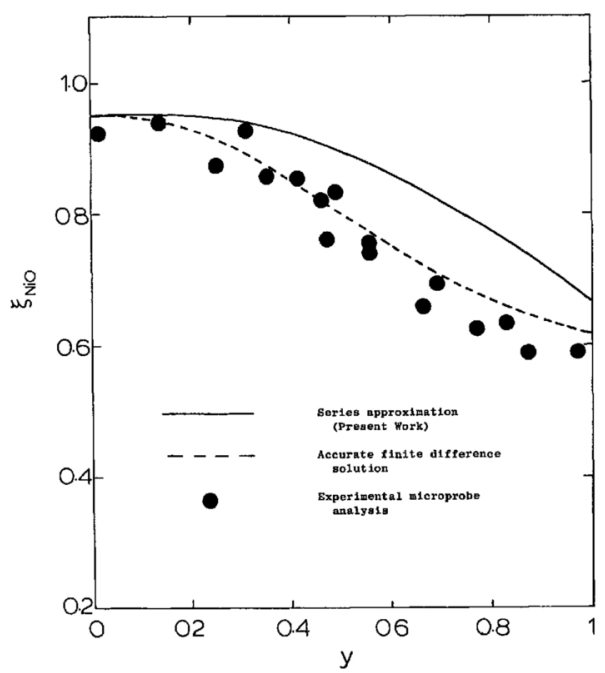

Fig. 3 Experimental and theoretical $\mathrm{Ni}$ profiles in the scale formed on a concentrated, Co $80 \mathrm{wt} \% \mathrm{Ni}$ alloy. At this concentration the error function approximation, eq. (30), is invalid and only the finite series approximation and the accurate finite difference analysis can be used to calculate cation profiles. function approximation (eq. (37)) can be used to describe cation profiles in scales on $\mathrm{Ni}$ rich alloys although accurate finite difference calculations continue to provide a more reliable result, as shown in Fig. 3 for a $\mathrm{Co}-$ $80 \% \mathrm{Ni}$ alloy.

\section{Conclusions}

(1) It has been demonstrated that the equations describing the distribution of cations in solid solution oxide scales can be solved analytically if it is assumed that the cation diffusivities are not dependent on the scale composition. The oxygen activity dependence of the diffusivities is included in the treatment and no restriction is placed on the relative concentrations of the cations in the scale. This contrasts with an earlier analysis in which it was necessary to assume that the diffusivities were independent of both the composition and the anion activity in the scale and also that the scale was dilute in one of the cation components. It is shown that this previously published expression is invalid when the scale or bulk alloy composition exceeds a certain limit and this limit is defined.

(2) The expression derived in the present work is obtained as a finite series if a simple defect lattice is assumed with only integral defect charges present. Otherwise the series is an infinite Taylor expansion although it is considered that the discrepancies between this general result and the simpler, finite series will be relatively small and that the latter can generally be used.

(3) Comparisons between the finite series and the previously published error function expressions are made for dilute $\mathrm{Co}-\mathrm{Ni}$ alloys and it is shown that they result in cation profiles which are essentially equivalent.

(4) In more concentrated alloys, close to the limit at which the error function expression becomes invalid, it is shown that the finite series provides a much more reliable description of the cation profile in the scale although this is not as reliable as an accurate finite difference solution of the relevant equations.

(5) For alloy concentrations which exceed the limit of validity of the error function ex- 
pression the finite series continues to provide a moderately accurate description of the cation profile in the scale although a complete finite difference solution of the relevant differential equations still results in the most accurate cation profile.

(6) A modification to the error function solution is suggested.

\section{Acknowledgment}

The authors would like to thank the Science Research Council for the financial support of this work.

\section{REFERENCES}

(1) C. Wagner: Corros. Sci., 9 (1969), 91.

(2) D. E. Coates and A. D. Dalvi: Oxid. of Metals, 2 (1970), 331.

(3) A. D. Dalvi and D. E. Coates: ibid., 3 (1971), 203.
(4) G. C. Wood and J. M. Ferguson: Nature, Lond., 208 (1965), 369.

(5) A. D. Dalvi and W. W. Smeltzer: J. Electrochem. Soc., 121 (1974), 386.

(6) P. Mayer and W. W. Smeltzer: ibid., 538.

(7) T. Narita and K. Nishida: J. Japan Inst. Metals, 29 (1975), 1152 (English translation kindly provide by authors).

(8) D. P. Whittle, B. D. Bastow and G. C. Wood: Oxid. of Metals, 9 (1975), 215.

(9) B. D. Bastow, D. P. Whittle and G. C. Wood: Corros. Sci., 16 (1976), 57.

(10) B. D. Bastow, D. P. Whittle and G. C. Wood: submitted to Proc. Roy. Soc.

(11) T. Narita and K. Nishida: Denki Kagaku, 43 (1975), 443.

(12) D. P. Whittle, D. J. Young and W. W. Smeltzer: J. Electrochem. Soc., 123 (1976), 1073.

(13) W. K. Chen and N. L. Peterson: J. Phys. Chem. Solids, 34 (1973), 1093.

(14) K. Nishida and T. Narita: Trans. JIM, 16 (1975), 755. 